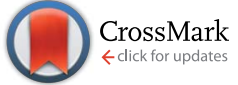

Cite this: J. Mater. Chem. A, 2016, 4, 14284

Received 1st August 2016

Accepted 15th August 2016

DOI: $10.1039 / c 6 t a 06573 b$

www.rsc.org/MaterialsA

\section{Synthesis of modified fullerenes for oxygen reduction reactions $\uparrow$}

\author{
Rosa María Girón, ${ }^{a}$ Juan Marco-Martínez, ${ }^{a}$ Sebastiano Bellani, ${ }^{\text {bc }}$ Alberto Insuasty, ${ }^{a}$

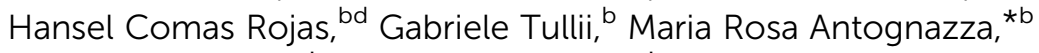 \\ Salvatore Filippone*a and Nazario Martín*ae
}

\begin{abstract}
The oxygen reduction reaction (ORR) is a key process common in several energy converting systems or electro-chemical technologies such as fuel cells, metal-air batteries, oxygen sensors, etc., which is based on the use of expensive and scarcely available platinum metal. In the search for carbon-based catalysts for ORRs, two different classes of new fullerene hybrids and metal-free fullerene derivatives endowed with suitable active sites have been prepared by highly selective metal- and organo-catalyzed synthetic methodologies. Along with their classical behavior as electron acceptors in polymer-based photo-electrochemical cells, the new fullerene derivatives are able to efficiently catalyze ORRs by using no metals or very low amounts of metals. Remarkably, the activity of metal-free fullerenes has proved to be as high as that observed for metallofullerenes bearing noble metals, and up to ten-fold higher than that of PCBM.
\end{abstract}

\section{Introduction}

The oxygen reduction reaction (ORR) is a key process in different energy converting systems or electrochemical technologies (fuel cells, metal air batteries, oxygen sensors, etc.). ${ }^{1}$ In these fields the replacement of the traditional platinum based catalysts with non-precious metals ${ }^{2}$ or metal-free electrocatalysts is currently a hot scientific challenge. ${ }^{3}$ Particularly, the use of carbon nanomaterials such as nitrogen-doped carbon nanotubes ${ }^{4}$ or graphene, ${ }^{5}$ represents an interesting and novel approach.

In this context, among the different allotropic forms of carbon, fullerenes combine unique chemical, ${ }^{6}$ optical, ${ }^{7}$ electronic $^{8}$ and photophysical ${ }^{9}$ properties with a defined molecular structure. Indeed, their good electron accepting and transport

\footnotetext{
${ }^{a}$ Departamento de Quimica Orgánica I, Facultad de Ciencias Quimicas, Universidad Complutense de Madrid, Ciudad Universitaria s/n, 28040 Madrid, Spain. E-mail: salvatorefilippone@ucm.es; nazmar@quim.ucm.es

${ }^{b}$ Center for Nano Science and Technology@PoliMi, Istituto Italiano di Tecnologia, Via Pascoli 70/3, 20133 Milano, Italy. E-mail: mariarosa.antognazza@iit.it

'Istituto Italiano di Tecnologia, Graphene Labs, Via Morego 30, Genova 16163, Italy ${ }^{d}$ Higher Institute for Applied Sciences and Technologies (INSTEC), Salvador Allende y Luaces, 6163, La Habana, Cuba

eIMDEA-Nanociencia, Campus de la Universidad Autónoma de Madrid, 28049 Madrid, Spain

$\dagger$ Electronic supplementary information (ESI) available: Experimental details of the preparation of the fullerene derivatives, polymer blend absorption spectra, spectroscopic and chromatographic data for the characterization of compounds, linear scan voltammetry and Cyclic Voltammetry (CV) measurements. CCDC 960717. For ESI and crystallographic data in CIF or other electronic format see DOI: 10.1039/c6ta06573b
}

capability led to their extended use as suitable n-type materials,$^{10}$ widely employed in organic electronics and photovoltaic devices, ${ }^{11}$ including the most recent perovskite solar cells. ${ }^{12}$

Thus, bulk heterojunction (BHJ) organic photovoltaic cells (OPVs) are a notable application, which consist of p-type conjugated polymers (e.g. poly(3-hexylthiophene-2,5-diyl), P3HT) and n-type fullerene derivatives, $[6,6]$ phenyl- $\mathrm{C}_{61}$-butyric acid methyl ester (PCBM) being the most widely used. In a typical BHJ approach, a bi-continuous network is created, which allows the promotion of exciton dissociation processes and the achievement of a high power conversion efficiency. The next frontier in the use of acceptor/donor BHJs, based on PCBM, is the realization of photo-electrochemical cells, able to work in contact with aqueous and/or non-aqueous electrolytes upon visible light illumination. One notable example is the realization of efficient photocathodes for hydrogen production by photo-electrochemical water splitting. ${ }^{13,14}$ Recently, some of us have reported a high sensitivity, photo-electrochemical oxygen sensor based on an organic $\mathrm{BHJ}$ formed using a low band gap polymer (APFO-3) and PCBM, revealing photo-activity towards the ORR. ${ }^{15}$ In the proposed scheme, polymer excitation by visible light leads to the efficient generation of bounded charged species (excitons), which are promptly dissociated into free charges (polarons) by a highly efficient electron transfer process to the fullerene-based acceptor domain. We have recently reported that this process, typical of solid-state photovoltaic cells, occurs in the hybrid system with fully comparable dynamics and efficiency. ${ }^{16}$ Photogenerated holes are collected at the underlying fluorine-doped tin oxide (FTO) electrode, while electrons are efficiently transferred at the 


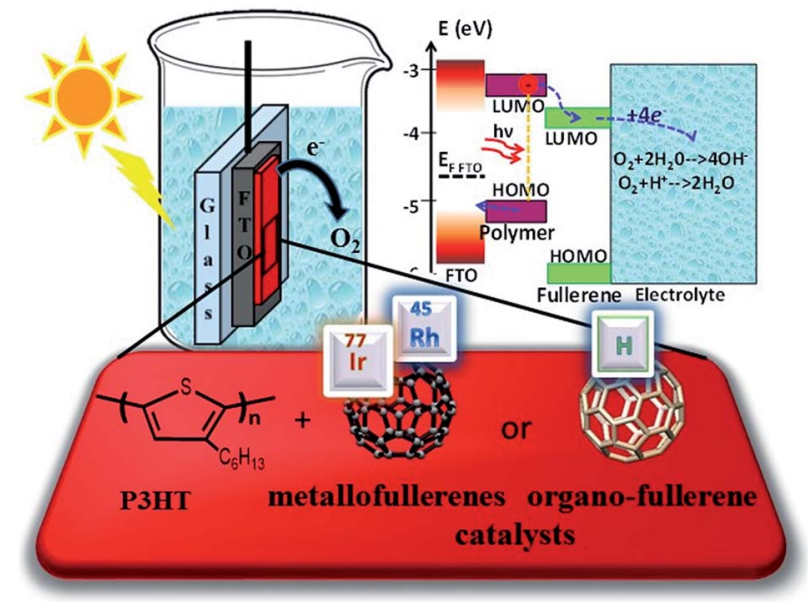

Fig. 1 Schematic sketch of an organic-based photo-electrochemical device for the ORR, based on P3HT:fullerene BHJ thin films. The working mechanism and the energy level schemes are also represented

interface with the aqueous solution, thus giving rise to photoelectrochemical reactions and, in particular, to the ORR (Fig. 1).

Here, we report the synthesis of new fullerene derivatives, suitably functionalized with catalytically active sites towards the ORR. This allows us to exploit a doubled fullerene functionality, by employing them both as electron acceptors in P3HT-based $\mathrm{BHJ}$, and as redox active sites, thus avoiding the use of expensive noble metals.

Two main approaches have been undertaken in parallel: (i) the synthesis of new fullerene hybrid derivatives endowed with noble metals active in redox processes, such as Ir, Rh or Pt; (ii) the synthesis of metal-free fullerene catalysts, based on the presence of active $\mathrm{C}_{60}-\mathrm{H}$ bonds. To this aim, both metal- and organo-catalytic methodologies have been employed, to obtain fullerene derivatives with tailored electronic and photocatalytic properties characterized by high regio- and stereo-selectivity.

Photoelectrochemical devices for the ORR based on $\mathrm{BHJ}$ P3HT:fullerene thin films have been finally realized, confirming an enhanced photocatalytic property of the novel compounds, when compared with the extensively used PCBM, taken as the reference fullerene.

\section{Results and discussion}

\subsection{Synthesis of metallo- and organo-fullerenes catalysts}

Over the last few years, fullerene chemistry has been widening its synthetic tools, in the search for more sophisticated structures, ${ }^{17}$ for a better control on the stereochemistry of the fullerene derivatives ${ }^{\mathbf{1 8}}$ and for a control on the regio- and siteselectivity when higher fullerenes ${ }^{19}$ or endohedral fullerenes ${ }^{20}$ are used. Following this trend, on one hand, we have directed our attention to the achievement of new selective catalytic methodologies aimed at the preparation of both metallofullerenes and regiopure fullerene hydrides. On the other hand, we have modified both metal- and organo-catalysed methodologies, ${ }^{21}$ previously described by us, for the introduction of a suitable functionality on the fullerene cage to become catalytically active towards the ORR.

2.1.1. Pyrrolino-metallo-fullerenes. With the aim of preparing stable metallo-fullerene hybrids, we first directed our attention onto iridium(III) complexes due to their wide use in hydrogenation processes and for their ability to form stable and easily isolable complexes. Thus, the design of the iridiumfullerene complexes was based on the preparation of pyrrolino [60]fullerene ligands endowed with a carboxylic group to bind iridium(III) as well as other active metals efficiently. To this purpose, we carried out the 1,3-dipolar cycloaddition reaction of azlactone 1 with [60]fullerene by using a racemic silver BINAP catalyst, followed by the addition of the iridium dimer $\left[\mathrm{Cp} \mathrm{IrCl}_{2}\right]_{2}$ (see Scheme 1). A unique compound, 2Ir, was obtained in "one-pot" and in good yield (ca. 40\%) as a result of two sequential processes where two chiral centers are formed: in the first step, a pyrrolino[3,4:1,2][60]fullerene carboxylic acid is formed with a stereogenic center in the C-5 of the pyrroline ring. In the second process, iridium(III) is covalently linked diastereoselectively to the [60]fullerene derivative through the formation of two new bonds, one with the nitrogen of the pyrroline ring and the other one with the oxygen of the carboxylate group. The presence of the metal is confirmed by the IR spectrum of the isolated solid, since the $\nu(\mathrm{CO})$ of the starting pyrroline shifts from $1700 \mathrm{~cm}^{-1}$ to $1660 \mathrm{~cm}^{-1}$ in the final product, which is in agreement with the previously observed behaviour of fullereneiminocarboxylate as (N,O)-chelating monoanionic ligands. ${ }^{22}$ It is worth noting that, despite iridium(III), with its typical pseudotetrahedral geometry, being able to adopt two possible configurations and, therefore, afford a diastereomeric mixture, the overall process occurs diastereoselectively, in sharp contrast to other related examples. ${ }^{23}$

Indeed, this behaviour was observed by ${ }^{1} \mathrm{H}$-NMR (see the ESI $\dagger$ ) and its structure could be confirmed by X-ray diffraction analysis of a monocrystal obtained by slow evaporation of 2 Ir in $\mathrm{CS}_{2} /$ hexane. $^{24}$

As is shown in Fig. 2, the iridium atom adopts a pseudooctahedral geometry where the $\mathrm{Cp}^{*}$ group occupies a face of the octahedron, $1.775 \AA$ being the distance between the metal and the ring centroid. Two other octahedral positions involve the chelate pyrrolinofullerene carboxylate featuring $\mathrm{Ir}-\mathrm{N}$ and $\mathrm{Ir}-\mathrm{O}$ bonds of 2.103(4) Å and 2.095(4) A length, respectively. Finally, the determined $\mathrm{Ir}-\mathrm{Cl}$ bond distance resulted to be 2.393(2) $\AA$,

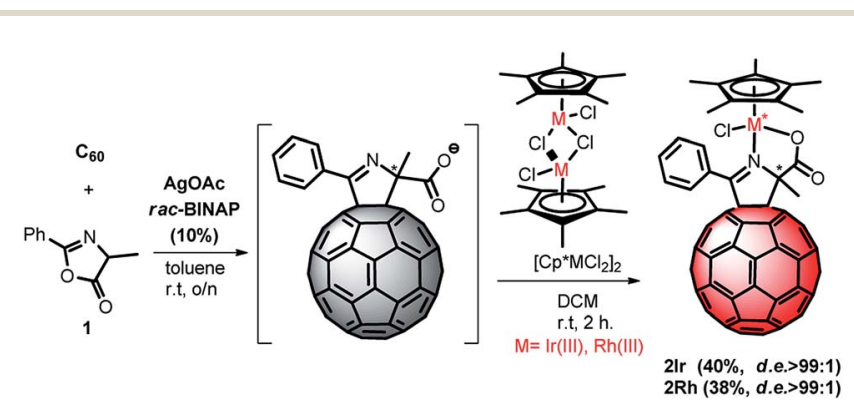

Scheme 1 Diastereomeric synthesis of iridium (2/r) and rhodium (2Rh) pyrrolino[3,4:1,2][60]fullerene. 

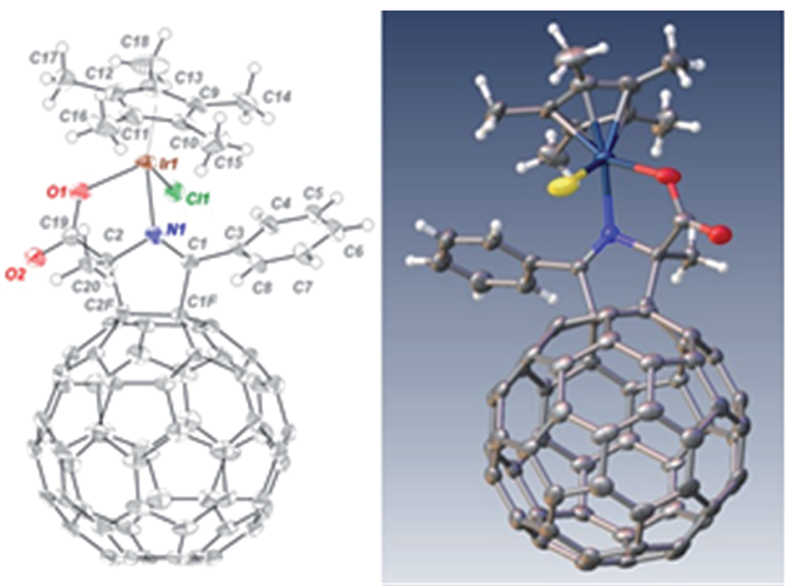

Fig. 2 X-ray diffraction of a crystal of 2 Ir obtained by slow evaporation in $\mathrm{CS}_{2}$ /hexane.

which is slightly shorter than that of other related pyrrolidinocarboxylate iridium $\mathrm{Cp}$ * complexes. ${ }^{23 b}$ The distance between the two fullerene $\mathrm{sp}^{3}$ carbon atoms is $1.590(8) \AA$, which is in the typical range for a fullerene monoadduct.

$\mathrm{X}$-ray analysis confirms the presence of a single diastereomer (with both enantiomers) with the chlorine atom in a trans position to the methyl group of the iridium cycle (see also ESI $\dagger$ ).

Analogously, a rhodium pyrroline[60]fullerene hybrid $\mathbf{2 R h}$ was prepared in $38 \%$ yield, by using $\left[\mathrm{Cp} * \mathrm{RhCl}_{2}\right]_{2}$ after the cycloaddition of the same azlactone 1 (Scheme 1).

2.1.2. Organocatalytic synthesis of the metal-free fullerene catalyst. Fullerenes with their 30 (for $\mathrm{C}_{60}$ ) or more strained $\mathrm{C}=\mathrm{C}$ double bonds have been envisaged as very useful molecular species for hydrogen storage and, therefore, fullerene hydrides (or fulleranes) have been deeply studied. ${ }^{25,26}$ Furthermore, these species easily undergo dehydrogenation in the presence of even very low amount of molecular oxygen. ${ }^{27}$ In this regard, we wondered if the presence of highly active hydrogen directly linked to the carbon cage could replace precious metals but still maintain a high catalytic activity. Thus, we designed a regioselective double addition on [60]fullerene where a $\mathrm{C}_{60}-\mathrm{H}$ bond is formed by the use of the organocatalytic methodology. To this aim, we extended the scope of our previously reported phosphine catalyzed cycloaddition of allenoates/alkynoates to [60] fullerene $e^{21 b, 28}$ to 5-hydroxy-3-alkynoates (Scheme 2).

Thus, after alkynoate/allenoate isomerization, 1,2-diphenylphosphino ethane (dppe) catalysed Lu's [3+2] cycloaddition ${ }^{29}$

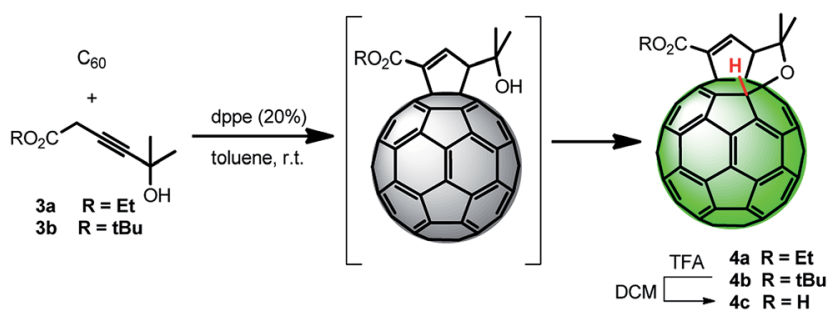

Scheme 2 cis-1 regioselective fullerene bifunctionalization affording fullerene hydrides. of $3 \mathbf{a}, \mathbf{b}$ afforded the corresponding cyclopentenoate[60]fullerenes. These products are not isolated since they underwent an easy regioselective addition of the hydroxyl group to the cis-1 double bond of $\mathrm{C}_{60}$ giving rise to bisadducts $\mathbf{4 a}, \mathbf{b}$ endowed with a $\mathrm{C}_{60}-\mathrm{H}$ bond in $48 \%$ and $34 \%$ yields, respectively. Eventually, the corresponding acid $\mathbf{4 c}$ was obtained after acidic removal of the tert-butyl ester.

\subsection{Photocatalytic activity of fullerene derivatives toward the ORR in photoelectrochemical devices}

The new fullerene derivatives were finally tested as photo-electrocatalysts in polymer-based devices for the photo-electrochemical ORR, and the overall electrochemical performances were compared to those of devices employing PCBM as a standard reference material. The compounds used are classified in Chart 1 into three main types: fullerenes without catalytic sites, but with higher-lying LUMO levels compared to PCBM, as mere acceptor components (DPM-12 and bisDPM-12, in grey colour, top panel); hybrid fullerenes with metallic catalytic sites (2Ir, $\mathbf{2 R h}$ and $\mathbf{6}$ compounds, in red) and catalytic, metal-free fullerenes (4a, $\mathbf{4 b}$ and $\mathbf{4 c}$ compounds, in green).

BHJ thin films $(\sim 140 \mathrm{~nm})$, based on rr-P3HT as a photoactive donor component, were deposited by spin coating on top of FTO-covered glass substrates. In some cases, a second thin film fullerene layer was deposited on top of the photoactive component, in order to directly expose the catalytic sites to the electrolyte. Photo-catalytic activity towards oxygen reduction was assessed in sodium phosphate buffer (PBS) at pH 7.4 and a controlled dissolved oxygen (DO) concentration $\left(5.8 \mathrm{mg} \mathrm{L}^{-1}\right)$. All the details about material absorbance spectra, device fabrication processing, experimental set up and measurement conditions are reported in the ESI section. $\dagger$

Fig. 3 reports Linear Scan Voltammetry (LSV) recorded in the dark and upon illumination ( 1 sun) in devices based on

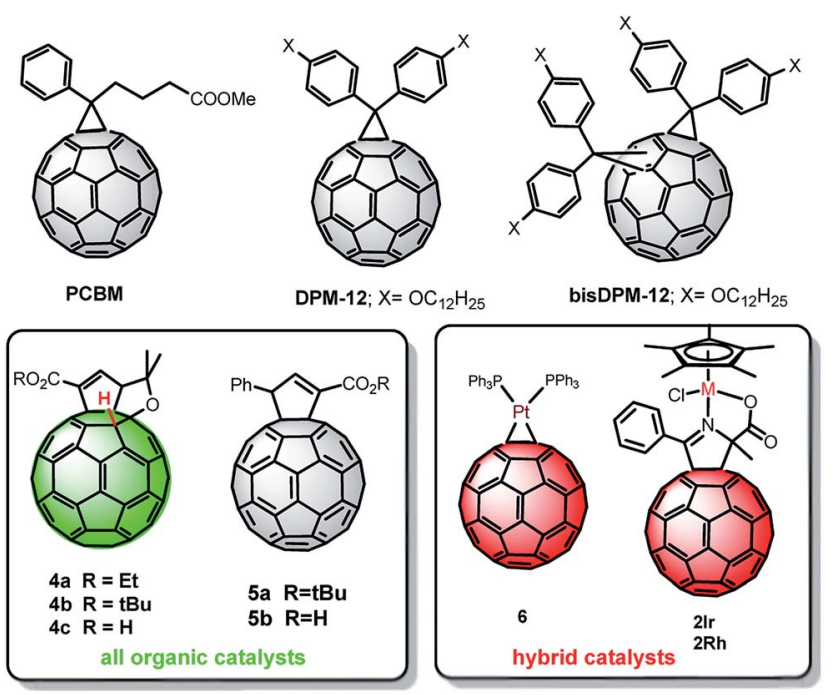

Chart 1 Fullerene derivatives used as acceptors/catalysts in photoelectrochemical devices. 


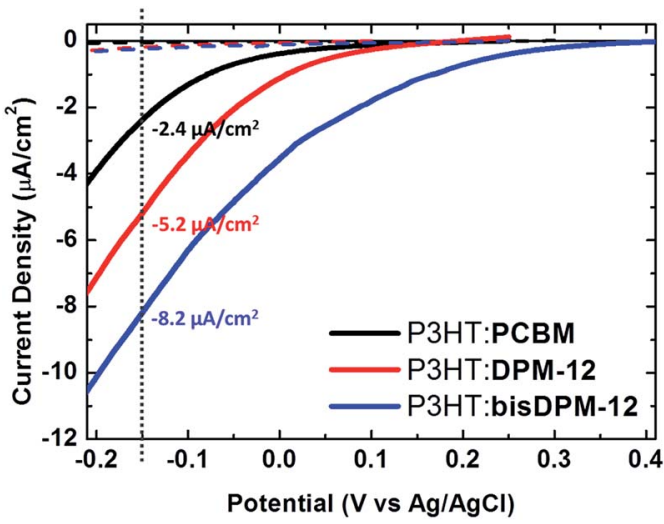

Fig. 3 Linear Scan Voltammetry (LSV) measurements in the dark (dashed lines) and upon visible light illumination (1 sun) (solid lines) on FTO/P3HT:fullerenes (PCBM, DPM-12 and bisDPM-12) for photoelectrochemical cells (scan rate, $10 \mathrm{mV} \mathrm{s}^{-1}$ ). Measurements were performed in sodium phosphate buffer (PBS) at $\mathrm{pH} 7.4$ and controlled dissolved oxygen (DO) concentration $\left(5.8 \mathrm{mg} \mathrm{L}^{-1}\right)$.

fullerene acceptors without any catalytic site (PCBM, DPM-12 and bisDPM-12).

As demonstrated in a previous work, ${ }^{15}$ the recorded current signal can be unambiguously attributed to photo-activated electrochemical reactions occurring at the hybrid organic/electrolyte interface and in particular to the ORR (see also Fig. S2 in the ESI section $\dagger$ ). Moreover, dark current values (dashed lines) are negligible in all cases.

Notably, the use of DPM-12 and bisDPM-12 fullerene bisadducts allows the increase of the Onset Potential (OP, defined here as the voltage to which the photocurrent density amounts at $100 \mathrm{nA} \mathrm{cm}{ }^{-2}$ ) up to $0.15 \mathrm{~V} v s$. $\mathrm{Ag} / \mathrm{AgCl}$ and $0.35 \mathrm{~V} v s$. $\mathrm{Ag} / \mathrm{AgCl}$, respectively, with respect to the reference PCBM, $0.12 \mathrm{~V} v s$. $\mathrm{Ag} / \mathrm{AgCl}$. Correspondingly, at a fixed voltage the photocurrent density increases: at $-0.15 \mathrm{~V}$ vs. $\mathrm{Ag} / \mathrm{AgCl}$, for instance, it amounts to $-2.4 \mu \mathrm{A} \mathrm{cm}^{-2},-5.2 \mu \mathrm{A} \mathrm{cm}^{-2}$, and $-8.2 \mu \mathrm{A} \mathrm{cm}^{-2}$ for PCBM, DPM-12 and bisDPM-12, respectively. An analogous behavior has also been reported in organic solar cells, where an increase of the open circuit voltage in the case of DPM-12 and bisDPM-12 was reported, when compared to PCBM. ${ }^{30}$ The enhanced performances are possibly attributed to the broader density of states and to the higher-lying LUMO level of the fullerene bisadducts, able to facilitate the electron transfer processes occurring at the organic/electrolyte interfaces.

Iridium, rhodium and platinum are known to be efficient catalysts for the ORR. ${ }^{31}$ Hybrid catalysts, 2 Ir, 2 Rh and 6, are thus expected to provide catalytic properties typical of the embedded metal component, but with a consistently reduced need for precious metals, and with the advantage of a more localized interaction. In this case photo-electrodes were realized by covering the reference P3HT:PCBM BHJ layer with an over-layer of pristine electron acceptor, in order to maximize the localization at the electrolyte interface of the catalytic sites present within the functionalized fullerene derivatives (P3HT:PCBM/ hybrid catalyst configuration).

The adopted strategy resulted to be successful. All tested hybrid catalysts show photocurrent density values larger by more than one order of magnitude with respect to reference PCBM, and comparable dark current values (Fig. 4). Photocurrent generation is clearly related to the presence of dissolved oxygen, and its origin can be safely attributed to the occurrence of the ORR (see ESI, Fig. S4, $\uparrow$ panels a and b). OPs are also considerably increased, shifting from $0.12 \mathrm{~V}$ vs. $\mathrm{Ag} / \mathrm{AgCl}$ for PCBM up to $0.3 \mathrm{~V} v s$. $\mathrm{Ag} / \mathrm{AgCl}$ for $2 \mathrm{Ir}$ and $2 \mathrm{Rh}$ and to $0.35 \mathrm{~V} v s$. $\mathrm{Ag} / \mathrm{AgCl}$ for 6. The reported data allow us to conclude that electrons can be easily transferred towards metallic catalytic centres which, in turn are highly efficient in reducing the overpotential needed to foster the ORR. In other words, the use of hybrid fullerenes endowed with catalytic centres increases both the electron transfer rate (observed as a net photocurrent density increase) and the driving force for electrochemical reactions (observed as an OP increase).

On the other hand, following the recent trend of using carbon based materials as the catalyst for the ORR,,$^{3-5}$ we planned the preparation of a molecular organocatalyst by replacement of the metallic atoms from the fullerene derivatives, with a fullerene hydride as the active site. The choice of using catalysts such as $\mathbf{4 a - c}$ relies on the reported hydrogen transfer from the fullerene hydrides, namely $\mathrm{C}_{60} \mathrm{H}_{2}$, to dioxygen ${ }^{27}$ and on the easy deprotonation of fullerene hydrides leading to very stable fullerene anions $\mathbf{4}^{-} .^{26}$

In order to evaluate the importance of the hydrogen fullerene bond, devices based on analogous cyclopentenoate functionalization but lacking the $\mathrm{C}_{60}-\mathrm{H}$ bond $(5 \mathbf{a}, \mathbf{b})$ were also prepared.

Fig. 5 summarizes the results of the characterization of photo-electrochemical cells based on $4 \mathbf{a}, \mathbf{4 b}, \mathbf{4 c}, 5 \mathbf{a}$ and $5 \mathbf{b}$ components as hybrid electron acceptor/catalyst components, as compared to the reference device based on PCBM.

We notice that 4 a leads to higher catalytic activity, with a more than 5-fold increase in photocurrent density at $-0.15 \mathrm{~V}$ vs. $\mathrm{Ag} / \mathrm{AgCl}$ with respect to $\mathrm{PCBM}$, and a higher OP value $(0.3 \mathrm{~V}$ vs. $\mathrm{Ag} / \mathrm{AgCl})$. Conversely, $\mathbf{5} \mathbf{a}$ and $\mathbf{5} \mathbf{b}$, despite presenting a similar

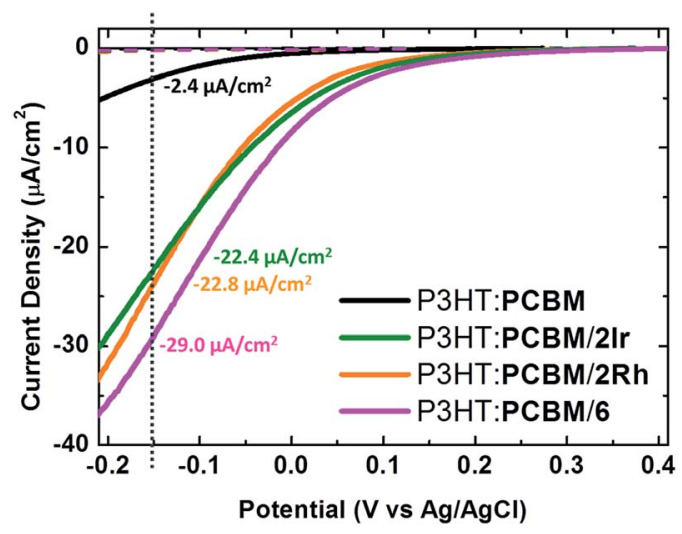

Fig. 4 Hybrid fullerenes, providing metallic catalytic sites, for the ORR. Linear Scan Voltammetry (LSV) measurements (scan rate, $10 \mathrm{mV} \mathrm{s}^{-1}$ ) in the dark (dashed lines) and upon visible light illumination (1 sun) (solid lines) on FTO/P3HT:PCBM/hybrid catalysts (2Ir, 2Rh and 6) for photoelectrochemical cells. Measurements were performed in sodium phosphate buffer (PBS) at pH 7.4 and controlled dissolved oxygen (DO) concentration $\left(5.8 \mathrm{mg} \mathrm{L}^{-1}\right.$ ). 


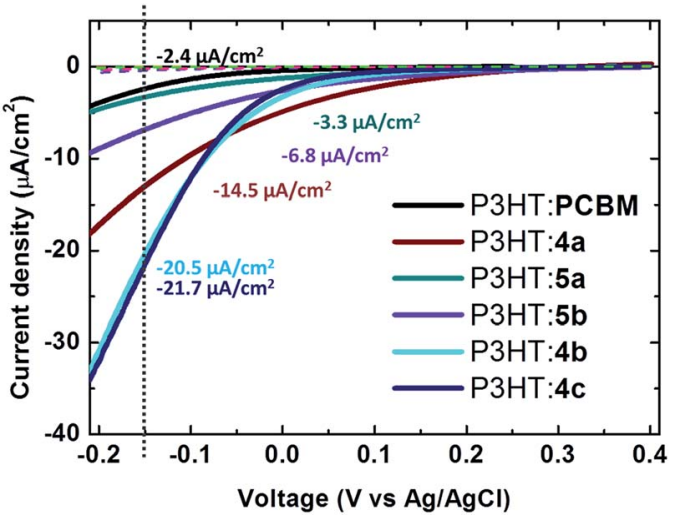

Fig. 5 Use of metal-free fullerene catalysts for the ORR. Linear Scan Voltammetry (LSV) measurements (scan rate, $10 \mathrm{mV} \mathrm{s}^{-1}$ ) in the dark (dashed lines) and upon visible light illumination (1 sun) (solid lines) on FTO/P3HT:fullerene catalysts (4a, 5a, 5b, $4 b$ and $4 c)$. Measurements were performed in sodium phosphate buffer (PBS) at pH 7.4 and controlled dissolved oxygen (DO) concentration $\left(5.8 \mathrm{mg} \mathrm{L}^{-1}\right)$.

cyclopentenoate moiety on the $\mathrm{C}_{60}$ cage, do not show a significant increase of the performance due to the lack of the $\mathrm{C}_{60}-\mathrm{H}$ bond. While 5a exhibits a similar behaviour to PCBM, the higher hydrophilicity of $\mathbf{5 b}$, bearing a carboxylic acid group, leads to a closer interaction with dissolved oxygen molecules, which could explain the higher photocurrent densities observed in $\mathbf{5 b}$.

Finally, we further functionalized $\mathbf{5 a}$ and $\mathbf{5 b}$ compounds by directly linking the active hydrogen to the carbon cage, as in the case of the more efficient $\mathbf{4 a}$ compound, obtaining $\mathbf{4 b}$ and $\mathbf{4 c}$, respectively. Once more, photocurrent generation is related to the occurrence of the ORR (see ESI, Fig. S4, panel c $\dagger$ ). Additional measurements under $\mathrm{O}_{2}$-saturated conditions on $\mathbf{4 b}$ and $\mathbf{4 c}$ are shown in the ESI (Fig. S5†). These data have been compared with those based on PCBM. Importantly, the recorded photocurrent densities for $\mathbf{4 b}$ and $\mathbf{4 c}$ show values comparable to the ones obtained for the hybrid catalysts P3HT:PCBM/2Ir and P3HT:PCBM/2Rh, in the order of $-20 \mu \mathrm{A} \mathrm{cm}^{-2}$ at $-0.15 \mathrm{~V} v \mathrm{~s}$. $\mathrm{Ag} /$ $\mathrm{AgCl}$. OPs are $0.28 \mathrm{~V}$ in both cases which are lower with respect to the ones of precious metal hybrid samples $(0.3 \mathrm{~V} v s . \mathrm{Ag} / \mathrm{AgCl}$ for PCBM/2Ir and $0.35 \mathrm{~V} v$ s. $\mathrm{Ag} / \mathrm{AgCl}$ for PCBM/2Rh and PCBM/ 6) suggesting the need for further improvement. Conversely, metal-free fullerene samples showed higher durability with respect to the precious metal hybrid samples. As a figure of merit, the photocurrents recorded at $-0.2 \mathrm{~V} v s$. $\mathrm{Ag} / \mathrm{AgCl}$ for consecutive Cyclic Voltammetry (CV) cycles have been taken (see ESI, Fig. S6 $\dagger$ ). At the fortieth cycle a decrease of the photocurrent of $12.9 \%, 21.2 \%$ and $45.4 \%$, with respect to the value recorded at the first cycle, is observed for the case of PCBM, $\mathbf{4 c}$ and 6, respectively. Interestingly, while for PCBM and $\mathbf{4 c}$ the photocurrent reached stable values after a few cycles of stabilization, in the case of $\mathbf{6}$ a progressive decrease is observed. This could be due to different ORR mechanisms for the hybrid catalyst when compared to the other cases. As a matter of fact, for the Pt electrode, two-electron reduction of oxygen to $\mathrm{H}_{2} \mathrm{O}_{2}$ occurs parallel to the four-electron reduction to $\mathrm{H}_{2} \mathrm{O}$ (see ESI,

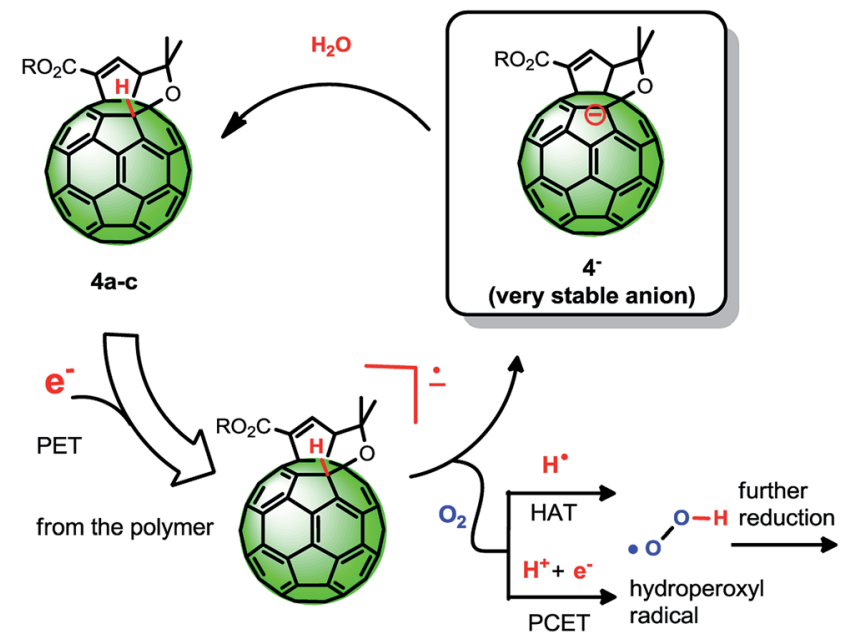

Scheme 3 Plausible ORR mechanism involving fullerene organocatalysts.

Scheme S1†). Consequently, a decrease of the cell voltage could arise because of the lower reversible redox potential of $\mathrm{H}_{2} \mathrm{O}_{2}$. Similar effects have been also reported in the durability studies of fuel cells. ${ }^{32}$

Regarding the catalytic activity of the metal-free fullerene derivatives $\mathbf{4 a - c}$, a plausible mechanism to account for the photocurrent increase and observed stability is based on the initial transfer of the photo-generated electron (PET) from the polymer to catalysts $\mathbf{4 a - c}$ affording the corresponding fullerene radical anion (Scheme 3). Thus, an easier oxygen reduction takes place to form the hydroperoxyl radical, through a hydrogen atom transfer (HAT) or by a proton coupled electron transfer (PCET), driven by the formation of the highly stable fullerene anions $\mathbf{4}^{-}$.

The aforementioned results show the remarkable activity of fullerene-based multiple metal-free catalysts in ORR, with efficiencies comparable to those obtained from metal-containing hybrid catalysts.

\section{Conclusions}

In summary, we have reported the highly selective catalytic synthesis of two series of fullerene-based molecular catalysts for the ORR. Iridium and rhodium pyrrolinofullerene complexes were prepared with complete diastereoselectivity (d.e. > 99\%) and the structure of the iridium-fullerene complex was confirmed by X-ray analysis. On the other hand, metal-free fullerene catalysts, endowed with a highly active $\mathrm{C}_{60}-\mathrm{H}$ bond, have been obtained by a regioselective cis-1 addition to the $\mathrm{C}_{60}$ cage. The electrocatalytic activity versus the oxygen reduction reactions has been tested in bulk heterojunction photo-electrochemical cells, affording current values up to ten fold higher than those of widely used PCBM. Remarkably, metal-free fullerene derivatives proved to give photocurrents comparable to those of related hybrids, thanks to the highly active $\mathrm{C}_{60}-\mathrm{H}$ bond on the fullerene cage. Having demonstrated that the approach is highly promising, future efforts will focus on 
further improvement of the overall performances of photoelectrochemical cells.

\section{Acknowledgements}

We acknowledge the financial support of the European Community through the Future and Emerging Technologies (FET) programme under the FP7, collaborative Project contract no. 309223 (PHOCS). NM thanks the European Research Council ERC-2012-ADG_20120216 (Chirallcarbon), MINECO of Spain (CTQ2014-52045-R) and the CAM (FOTOCARBON project S2013/MIT-2841). R. M. G. thanks Ministerio de Educación, Cultura y Deporte for the FPU contract.

\section{Notes and references}

1 (a) B. C. H. Steele and A. Heinzel, Nature, 2001, 414, 345-352; (b) F. Cheng and J. Chen, Chem. Soc. Rev., 2012, 41, 21722192.

2 Z. Chen, D. Higgins, A. Yu, L. Zhang and J. Zhang, Energy Environ. Sci., 2011, 4, 3167-3192.

3 (a) L. Dai, Y. Xue, L. Qu, H.-J. Choi and J.-B. Baek, Chem. Rev., 2015, 115, 4823-4892; (b) D.-W. Wang and D. Su, Energy Environ. Sci., 2014, 7, 576-591.

4 K. Gong, F. Du, Z. Xia, M. Durstock and L. Dai, Science, 2009, 323, 760-764.

5 (a) D. Higgins, P. Zamani, A. Yu and Z. Chen, Energy Environ. Sci., 2016, 9, 357-390; (b) J. Guan, X. Chen, T. Wei, F. Liu, S. Wang, Q. Yang, Y. Lu and S. Yang, J. Mater. Chem. A, 2015, 3, 4139-4146.

6 A. Hirsch and M. Brettreich, Fullerenes: Chemistry and Reactions, Wiley VCH, Weinheim, Germany, 2005.

7 L. W. Tutt and A. Kost, Nature, 1992, 356, 225-226.

8 L. Echegoyen and L. E. Echegoyen, Acc. Chem. Res., 1998, 31, 593-601.

9 D. M. Guldi and M. Prato, Acc. Chem. Res., 2000, 33, 695-703.

10 J. E. Anthony, A. Facchetti, M. Heeney, S. R. Marder and X. Zhan, Adv. Mater., 2010, 22, 3876-3892.

11 (a) J. L. Delgado, P.-A. Bouit, S. Filippone, M. A. Herranz and N. Martin, Chem. Commun., 2010, 46, 4853-4865; (b) B. C. Thompson and J. M. J. Fréchet, Angew. Chem., Int. Ed., 2008, 47, 58-77.

12 (a) A. Abrusci, S. D. Stranks, P. Docampo, H.-L. Yip, A. K. Y. Jen and H. J. Snaith, Nano Lett., 2013, 13, 31243128; (b) P.-W. Liang, C.-C. Chueh, S. T. Williams and A. K. Y. Jen, Adv. Energy Mater., 2015, 5, 1402321-1402328.

13 (a) F. Fumagalli, S. Bellani, M. Schreier, S. Leonardi, H. C. Rojas, A. Ghadirzadeh, G. Tullii, A. Savoini, G. Marra, L. Meda, M. Gratzel, G. Lanzani, M. T. Mayer, M. R. Antognazza and F. Di Fonzo, J. Mater. Chem. A, 2016, 4, 2178-2187; (b) T. Bourgeteau, D. Tondelier, B. Geffroy, R. Brisse, R. Cornut, V. Artero and B. Jousselme, ACS Appl. Mater. Interfaces, 2015, 7, 16395-16403; (c) M. Haro, C. Solis, G. Molina, L. Otero, J. Bisquert, S. Gimenez and A. Guerrero, J. Phys. Chem. C, 2015, 119, 6488-6494; (d) G. M. Suppes, P. J. Fortin and S. Holdcroft, J. Electrochem. Soc., 2015, 162, H551-H556; (e) M. P. Gustafson, N. Clark,
B. Winther-Jensen and D. R. MacFarlane, Electrochim. Acta, 2014, 140, 309-313.

14 H. Comas Rojas, S. Bellani, F. Fumagalli, G. Tullii, S. Leonardi, M. T. Mayer, M. Schreier, M. Grätzel, G. Lanzani, F. Di Fonzo and M. R. Antognazza, Energy Environ. Sci., 2016, DOI: 10.1039/c6ee01655c.

15 S. Bellani, A. Ghadirzadeh, L. Meda, A. Savoini, A. Tacca, G. Marra, R. Meira, J. Morgado, F. Di Fonzo and M. R. Antognazza, Adv. Funct. Mater., 2015, 25, 4531-4538.

16 A. Guerrero, M. Haro, S. Bellani, M. R. Antognazza, L. Meda, S. Gimenez and J. Bisquert, Energy Environ. Sci., 2014, 7, 3666-3673.

17 N. Martín, M. Altable, S. Filippone and A. Martín-Domenech, Synlett, 2007, 3077.

18 E. E. Maroto, M. Izquierdo, S. Reboredo, J. Marco-Martínez, S. Filippone and N. Martín, Acc. Chem. Res., 2014, 47, 26602670.

19 E. E. Maroto, A. de Cózar, S. Filippone, Á. Martín-Domenech, M. Suarez, F. P. Cossío and N. Martín, Angew. Chem., Int. Ed., 2011, 50, 6060-6064.

20 (a) E. E. Maroto, J. Mateos, M. Garcia-Borràs, S. Osuna, S. Filippone, M. Á. Herranz, Y. Murata, M. Solà and N. Martín, J. Am. Chem. Soc., 2015, 137, 1190-1197; (b) E. E. Maroto, M. Izquierdo, M. Murata, S. Filippone, K. Komatsu, Y. Murata and N. Martín, Chem. Commun., 2014, 50, 740-742; (c) K. Sawai, Y. Takano, M. Izquierdo, S. Filippone, N. Martín, Z. Slanina, N. Mizorogi, M. Waelchli, T. Tsuchiya, T. Akasaka and S. Nagase, J. Am. Chem. Soc., 2011, 133, 17746-17752.

21 (a) J. Marco-Martínez, S. Reboredo, M. Izquierdo, V. Marcos, J. L. López, S. Filippone and N. Martín, J. Am. Chem. Soc., 2014, 136, 2897-2904; (b) J. Marco-Martínez, V. Marcos, S. Reboredo, S. Filippone and N. Martín, Angew. Chem., Int. Ed., 2013, 52, 5115-5119.

22 (a) S. H. Laurie, in Comprehensive Coordination Chemistry, ed. G. Wilkinson, Pergamon, Oxford, 1987, vol. 2, ch. 20.2, p. 739; (b) K. Nakamoto, Infrared and Raman Spectra of Inorganic and Coordination Compounds, Wiley, New York, 4th edn, 1986, p. 233.

23 (a) W. Bauer, M. Prem, K. Polborn, K. Sünkel, W. Steglich and W. Beck, Eur. J. Inorg. Chem., 1998, 1998, 485-493; (b) D. Carmona, M. Pilar Lamata, F. Viguri, E. San José, A. Mendoza, F. J. Lahoz, P. García-Orduña, R. Atencio and L. A. Oro, J. Organomet. Chem., 2012, 717, 152-163.

24 See the ESI $\dagger$

25 Fulleranes the Hydrogenated Fullerenes, ed., F. Cataldo and S. Iglesias-Groth, Springer Science+Business Media B.V., Dordrecht, 2010.

26 J. Nossal, R. K. Saini, L. B. Alemany, M. Meier and W. E. Billups, Eur. J. Org. Chem., 2001, 4167-4180.

27 G. W. Wang, Y. J. Li, F. B. Li and Y. C. Liu, Lett. Org. Chem., 2005, 2, 595-598.

28 R. M. Girón, S. Reboredo, J. Marco-Martínez, S. Filippone and N. Martín, Faraday Discuss., 2014, 173, 311-322.

29 C. Zhang and X. Lu, J. Org. Chem., 1995, 60, 2906-2908.

30 (a) A. Sánchez-Díaz, M. Izquierdo, S. Filippone, N. Martin and E. Palomares, Adv. Funct. Mater., 2010, 20, 2695-2700; 
(b) G. Garcia-Belmonte, P. P. Boix, J. Bisquert, M. Lenes, H. J. Bolink, A. La Rosa, S. Filippone and N. Martín, J. Phys. Chem. Lett., 2010, 1, 2566-2571; (c) Y.-J. Cheng, M.-H. Liao, C.-Y. Chang, W.-S. Kao, C.-E. Wu and C.-S. Hsu, Chem. Mater., 2011, 23, 4056-4062.

31 (a) D. Cao, A. Wieckowski, J. Inukai and N. Alonso-Vante, J. Electrochem. Soc., 2006, 153, A869-A874; (b) Y. Nie, L. Li and Z. Wei, Chem. Soc. Rev., 2015, 44, 2168-2201; (c) J. Qiao, R. Lin, B. Li, J. Ma and J. Liu, Electrochim. Acta, 2010, 55, 8490-8497.

32 (a) J. Qiao, M. Saito, K. Hayamizu and T. Okada, J. Electrochem. Soc., 2006, 15, A967-A974; (b) V. A. Sethuraman, J. W. Weidner, A. T. Haug, M. Pemberton and L. V. Protsailo, Electrochim. Acta, 2009, 54, 5571-5582. 